\title{
Focus determination for the James Webb Space Telescope Science Instruments: A Survey of Methods
}

\author{
Pamela S. Davila*a ${ }^{*}$ Matthew R. Bolcar ${ }^{\mathrm{b}}$, Brent J. Bos, Bruce Dean, John G. Hagopian, Joseph M. \\ Howard, Blair Unger, Mark E. Wilson
}

${ }^{a}$ Goddard Space Flight Center, Code 551, Greenbelt Rd, Greenbelt, MD 20771

${ }^{\mathrm{b}}$ University of Rochester, Rochester, New York, 14611

\begin{abstract}
The James Webb Space Telescope (JWST) is a segmented deployable telescope that will require on-orbit alignment using the Near Infrared Camera as a wavefront sensor. The telescope will be aligned by adjusting seven degrees of freedom on each of 18 primary mirror segments and five degrees of freedom on the secondary mirror to optimize the performance of the telescope and camera at a wavelength of 2 microns. With the completion of these adjustments, the telescope focus is set and the optical performance of each of the other science instruments should then be optimal without making further telescope focus adjustments for each individual instrument. This alignment approach requires confocality of the instruments after integration and alignment to the composite metering structure, which will be verified during instrument level testing at Goddard Space Flight Center with a telescope optical simulator. In this paper, we present the results from a study of several analytical approaches to determine the focus for each instrument. The goal of the study is to compare the accuracies obtained for each method, and to select the most feasible for use during optical testing.
\end{abstract}

Keywords: James Webb Space Telescope, telescope, science instruments, focus

\section{INTRODUCTION}

Figure 1 is an artist's conception of the James Webb Space Telescope Observatory. The Observatory architecture

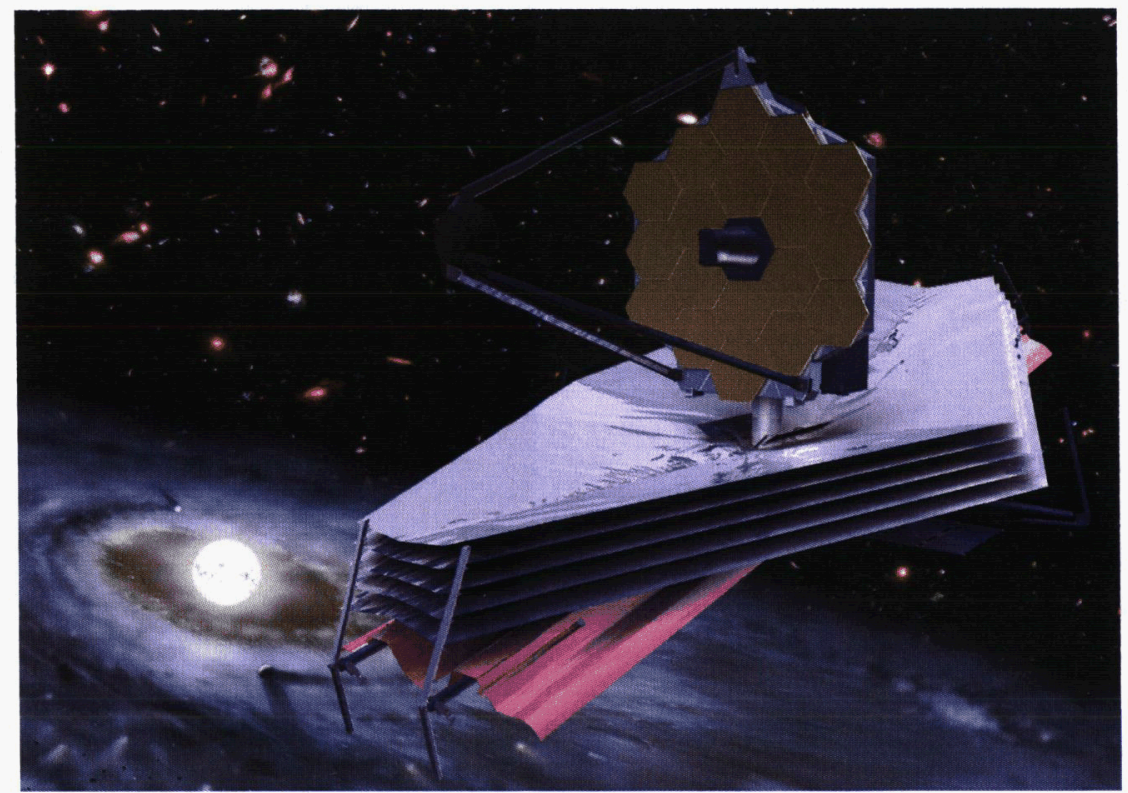

Figure 1. The JWST Observatory.

\footnotetext{
*Pamela.S.Davila@nasa.gov, Phone 1301 286-2074 ; Fax 1301 286-0204
} 
consists of a deployable Optical Telescope Element (OTE) with a $6.6 \mathrm{~m}$ diameter, segmented, primary mirror that is passively cooled to $30-50 \mathrm{~K}$ at the thermally stable L2 LaGrange point orbit. The curved focal surface from the telescope is located within the mechanical volume allocated to the Integrated Science Instrument Module (ISIM). The ISIM structure, the enclosure, the suite of instruments and guider and the other auxiliary hardware such as electrical harnesses and thermal devices and the kinematic mounts that interface the ISIM to the telescope is called the Integrated Science Instrument Module. The ISIM structure consists of a composite metering structure designed to interface by way. of kinematic mounts to each of the optical benches of the science instruments and the guider. The structue is also kinematically mounted to the telescope primary mirror structure. An enclosure surrounds the ISIM structure, isolates the ISIM region thermally from the other regions of the Observatory, and serves as a radiator for the science instruments and guider. (Note: The ISIM and enclosure are located behind the primary mirror in Figure 1). Light from the OTE is directed toward the instrument apertures by individual instrument pick-off mirrors located along the OTE light path. The four Science Instruments (SIs) are: The Near-InfraRed Camera (NIRCam), the Near-InfraRed Spectrometer (NIRSpec), the Mid-InfraRed Instrument (MIRI), and the Fine Guidance Sensor (FGS) which consists of both a Guider and a Tunable Filter (TF). During on-orbit commissioning, the telescope focus will be set as a result of the wavefront sensing and control process using the NIRCam. On-orbit adjustment of the telescope focus to optimize the performance of each individual SI before a scheduled science observation is not planned.

The science instruments and guider will be integrated to the ISIM structure and optically tested at Goddard Space Flight Center with an OTE SIMulator, or OSIM. The OSIM will be used for optical performance testing of the Engineering Test Unit (ETU) ISIM and SIs and the Flight ISIM and SIs and FGS. These tests will be conducted in the Space Environmental System (SES) thermal vacuum chamber at Goddard. The science instruments must be aligned in the test configuration to be confocal meaning "with the same focus."

One of the more important objectives of this study is to compare and contrast various methods used for the determination of best focus. In section 2 we discuss the optical models of the OTE and the SIs. In section 3 we describe the modeling process and in Section 4 we describe the focus methods considered. Finally, in Section 5 we summarize the results.

\section{CODE V MODELS OF THE SCIENCE INSTRUMENTS AND GUIDER}

Table 1 lists the filenames of the Code V models we used for the SIs, Guider and OTE. Figure 3 shows a Code V layout of the instrument designs. We excluded the Guider from this study since that instrument was being redesigned. At the time of this work, we analyzed the integrated OTE and SI models.

Table 1. Code V Filenames for the OTE and the SI optical models.

\begin{tabular}{|l|l|}
\hline OTE & OTE_Monolith_Rev_B \\
\hline NIRCam & SO501044.seq (short wavelength channel) \\
\hline & L0501044.seq_(long wavelength channel) \\
\hline NIRSpec & OTE_NIRS_A1_V2_MIRROR_cold.len \\
\hline & OTE_NIRS_A1_V2_MIRROR_cold.seq \\
\hline MIRI & Imager_mode1.len \\
\hline
\end{tabular}




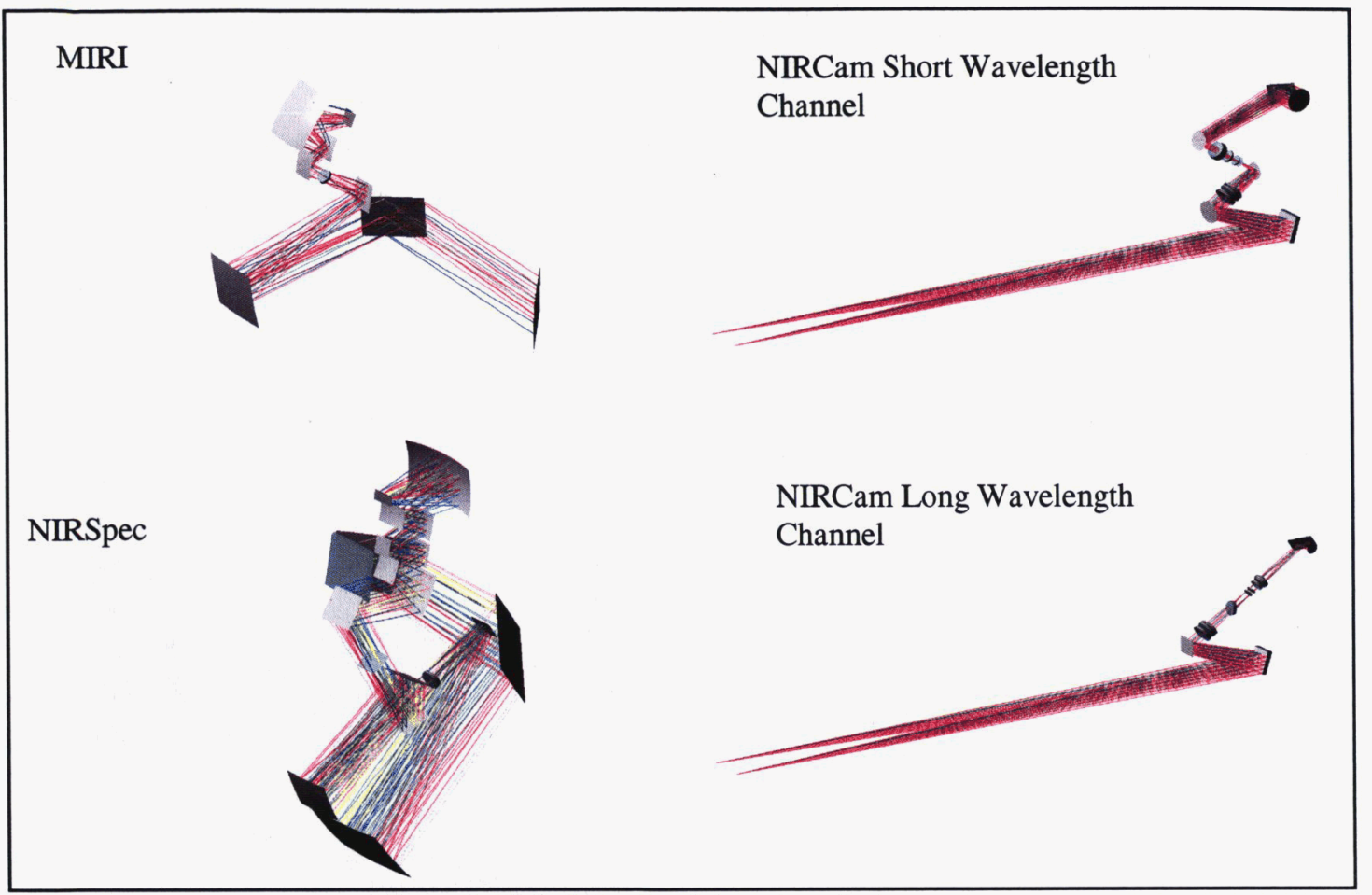

Figure 2. Optical layouts of the science instruments.

\section{THE MODELING PROCESS}

\subsection{Noise-Free Data}

For each optical model of the OTE and SI, a set of through-focus Point Spread Functions (PSF) was generated. The defocus occurred at the image surface of the OTE and in the direction along the chief ray at the field point under consideration for the particular instrument. In this way, a "pure" focus error was introduced with no lateral shift allowed between the OTE and each SI. The PSFs were generated at a single field point for each SI, corresponding to the center of an individual detector. For each simulation, PSFs were generated over a range of OTE defocus values from $+5 \mathrm{~mm}$ to -5 $\mathrm{mm}$ in steps of $0.5 \mathrm{~mm}$. For each simulation, a total of 21 data sets was created in X, Y, Z Matlab format with X, Y corresponding to detector pixel coordinates and $\mathrm{Z}$ corresponding to intensity on the detector at the pixel location. The PSF intensity values were normalized to the Strehl Ratio for each data set. The PSF data was oversampled for each detector pixel by a factor of five. Data was later rebinned for the appropriate detector pixel size. The simulated PSFs were polychromatic with the wavelengths and bandwidths indicated in Table 2 . Uniform spectral weighting was assumed with 5 wavelengths to cover the band. Detector pixel sizes for each instrument are also included in Table 2 .

To ensure that the quality of the focus metric is being evaluated and not the quality of the interpolation scheme, a Matlab algorithm was used as a common interpolation algorithm to determine the best focus. If a function is sufficiently sampled, then perfect interpolation can be achieved by convolving the sampled function with $\sin (\pi \mathrm{x}) / \pi \mathrm{x}$. But, due to the infinite extent of the sinc function, better results are obtained in-practice by using approximations to the sinc. Park and Schowengerdt ${ }^{1}$ (1983) have shown that a parametric cubic approximation to the sinc yields the optimum results and we used the version of this approach available in Matlab.

The data sets were analyzed using the methods described in Section 4. 
Table 2. List of modeling parameters for the various instruments.

\begin{tabular}{|c|c|c|c|c|c|c|c|}
\hline & $\begin{array}{c}\text { Center } \\
\lambda(\mathrm{um})\end{array}$ & Bandpass & $\begin{array}{c}\text { Pixel } \\
\text { size } \\
(\mathrm{um})\end{array}$ & $\begin{array}{c}\text { Field-X } \\
\text { (arcminutes) }\end{array}$ & $\begin{array}{c}\text { Field-Y } \\
\text { (arcminutes) }\end{array}$ & $\begin{array}{c}\text { \# of Detector } \\
\text { Pixels }\end{array}$ & f/ratio \\
\hline $\begin{array}{c}\text { NIRCam } \\
\text { short }\end{array}$ & 2.12 & $\mathrm{R}=5$ & 18 & 0.014930556 & 0.015291667 & $2048 \times 2048$ & 17.7 \\
\hline $\begin{array}{c}\text { NIRCam } \\
\text { long }\end{array}$ & 3.00 & $0.4 \mathrm{um}$ & 18 & 0.014930556 & 0.015291667 & $2048 \times 2048$ & 8.65 \\
\hline NIRSpec & 3.17 & & 18 & 0.10539 & 0.11913 & $2048 \times 2048$ & 5.37 \\
\hline MIRI & 5.70 & $1.2 \mathrm{um}$ & 25 & -0.005411 & 0.0 & $1024 \times 1024$ & 7.01 \\
\hline
\end{tabular}

For the purpose of comparison of each metric against a standard best focus value, Code $\mathrm{V}$ was used to calculate the best focus position for each SI using the criteria of minimum rms wavefront error at the specific field point of interest. This does not exactly match the criteria used during the design process for each instrument. For instance, in the case of NIRCam, the designer minimized the rms wavefront error over the field of view of the instrument resulting in a region of minimum rms around the middle of the field with larger excursions around the outer perimeter. During an actual measurement of focus for each instrument at GSFC, the data collection and analysis process will be more complex than our simple simulations here. For the focus verification test, the determination of best focus over the field of view of each SI will be determined using a minimum of five field points for each SI (one close to center and four near the edges or corners).

Table 3 lists the predicted Code $\mathrm{V}$ best focus values for each instrument. For comparison, we also show the minimum rms spot size best focus predictions.

Table 3. Predicted best focus values from Code $\mathrm{V}$ for each SI.

\begin{tabular}{|c|c|c|}
\hline & $\begin{array}{c}\text { Minimum RMS Spot Size } \\
\text { - predicted value from } \\
\text { Code V (mm of defocus } \\
\text { from nominal) }\end{array}$ & $\begin{array}{c}\text { Minimum RMS Wavefront } \\
\text { Error - predicted value from } \\
\text { Code V (mm of defocus from } \\
\text { nominal) }\end{array}$ \\
\hline NIRCam Long & 1.4922 & 1.58537 \\
\hline NIRCam Short & 0.07686 & 0.085758 \\
\hline NIRSpec & 0.29210 & 0.11583 \\
\hline MIRI & -0.60190 & -0.62625 \\
\hline
\end{tabular}

\subsection{Noisy Data}

A program called OPTOOL ${ }^{2}$ was used to add detector noise to the simulated PSFs. The user can select the focal plane array quantum efficiency, dark current, charge diffusion, and read noise; then detected images can be simulated. The input for this program is OPD data created using Code V. Along with OPD data, the vertical and horizontal f-numbers, entrance pupil diameter, and pupil mask geometry were also output. OPTOOL direct integration was used to calculate the PSFs with a grid spacing of 4.5 microns for the NIRCam and NIRSpec and 6.25 microns for MIRI, corresponding to a factor of 4 oversampling in all cases. Finally, detector effects were added and the PSFs were down sampled to their respective pixel sizes. Three different detector noise cases were run: (1) noisy $=$ Stellar Energy Distribution (SED) of 5, (2) noisier $=$ SED of 50 , and (3) noisiest $=$ SED of 5000 .

\section{A DESCRIPTION OF THE METHODS}

\subsection{High Pass Filter}

One method selected for study was reported by Price and $\mathrm{Gough}^{3}$ to output the most disparate signals between in-focus and out-of-focus scanning microscopy images. Bos ${ }^{4}$ acquired with Mars lander cameras. The approach is described mathematically in Equation 1 where $\mathrm{g}_{\mathrm{i}, \mathrm{j}}$ represents the image and ${ }^{* *}$ symbolizes a two-dimensional convolution. 
Inspection of Eq. 1 reveals that $F_{1}$ is the summation of a high pass filter operation. We investigated the performance of the high pass filter with and without the summation operation. One promising approach used the maximum pixel value produced by the high pass filtering operation.

$$
F_{1}=\sum_{i, j}\left(\left[\begin{array}{ccc}
-1 & -2 & -1 \\
-2 & 12 & -2 \\
-1 & -2 & -1
\end{array}\right] * * g_{i, j}\right)^{2}
$$

$F_{1}$ is the summation of a high pass filter operation. With the aid of equation (1), a single number indicative of the image's quality of focus can be determined for each out of focus image.

\subsection{Statistical Image Variance}

The statistical image variance is given by Equation (2):

$$
F_{2}=\frac{1}{n(n-1)}\left(n \sum_{i, j} g_{i, j}^{2}-\left(\sum_{i, j} g_{i, j}\right)^{2}\right)
$$

This equation yields another metric that can also be used to evaluate the quality of the focus.

\subsection{Peak Pixel}

This focus metric is simple and straightforward. For each out-of-focus image, the intensity is normalized to the peak value, and then the peak pixel value is plotted versus image number or focus position.

\subsection{Encircled Energy}

Encircled energy is a common metric used in the evaluation of system performance for astronomical telescopes. By measuring the ratio of energy contained within a small region around a PSFs center to the energy contained within an entire PSF, one can gain insight beyond a simple Strehl Ratio measurement. Encircled energy as a function of radial extent provides information about the distribution of low, mid, and high spatial frequencies in an image. Since encircled energy is one of the main performance metrics for JWST as well as being a valuable metric for understanding system performance in general, we chose to use it as one of the metrics for this study.

The simple encircled energy calculation used for this study has two main preprocessing steps: noise floor subtraction and PSF centering. The floor was assumed to be the mean of all data within a given PSF, and that value was subtracted from each numerical value in each PSF. The second operation was centering of the PSF centroid at the center of the image. Unfortunately, as the signal to noise ratio decreased and the signal approached the noise floor, the centroid of the PSF moved around sufficiently to affect the consistency of the encircled energy results.

The encircled energy algorithm performs two operations: upsampling and integration. For this study, the data was upsampled by four times using a bicubic interpolation scheme to fill in the upsampled data. This improves the subsequent integration since we have finer sampling at the edges. For small radii, the grid-to-circle conversion is more consistent when more pixels are used to approximate the circle. The integration itself is straightforward: sum up the energy contained within the entire PSF and sum up the energy contained within a specified radii. The ratio of those two 
sums yields a percent encircled energy. For noiseless data, encircled energy provides a consistent and reliable technique for determining defocus; however as noise increases, the encircled energy calculation diverges. A more robust data preprocessing could likely improve the performance of this metric for images with low SNR.

\subsection{Phase Retrieved Focus Values}

Phase-retrieval is an image-based wavefront sensing method that utilizes point source images to recover optical phase information. The most famous application of phase-retrieval was for the diagnosis of the Hubble Space Telescope mirror edge defect. ${ }^{5}$ Phase-retrieval is also the last step of the JWST optical commissioning process and is referred to as "finephasing." Details on the entire JWST commissioning process, as applied to the JWST Testbed Telescope, are discussed in these proceedings ${ }^{6}$. Results from the government fine-phasing algorithm as applied to data from the Testbed Telescope are also discussed in these proceedings?

A number of image-based phase-retrieval techniques have been developed that can be classified into one of two general categories: (a) iterative transform ${ }^{8}$ or (b) parametric. ${ }^{9,10}$ For the study considered in this paper, phase-retrieval was utilized to recover just a single optical parameter, defocus. As a result, it is often more efficient in such cases to implement a parametric approach by solving for the defocus value that minimizes the least-squares objective function:

$$
\chi^{2}=\sum_{i} \sum_{j} \mid d a t a_{i j}-\left.\bmod e l_{i j}(\text { defocus })\right|^{2}
$$

Various implementations of the parametric approach include minimizing alternative objective functions as well as implementing a variety of nonlinear optimization methods such as Levenburg-Marquardt, simplex, and quasi-Newton techniques. ${ }^{11}$ The Levenburg-Marquardt and quasi-Newton techniques were utilized in this study.

The Code V models of JWST were used to generate the OPDs while translating the focal-plane under various noise scenarios. The phase-retrieval algorithm was then applied to the leading and trailing images of each intensity data set, after registering the images to the center of the image array. A set of defocus values was then returned that minimizes the $\chi^{2}$ function defined in Equation (3) at each image location. The algorithm was not applied to images collected near focus since the estimation of the phase values near best focus is easily biased by spurious noise values. As a final step, the phase-retrieval defocus values (in waves) were fit using linear least squares to the measured defocus position (mm). The best focus position for each data set was then found by interpolation from the retrieved defocus values.

\section{SUMMARY OF RESULTS}

First we compared results from the focus methods using the noise-free, high-resolution NIRCam short-wavelength PSF's. The results obtained from the variance, peak pixel, encircled energy and high pass filter methods are shown in the Figure 3. The various color coded plots represent encircled energy diameters of $1,2,5,10$, and 20 pixels. From this comparison chart, several conclusions were drawn for the noise free simulations: (1) the method that represented the sharpest determination of focus was the high pass filter method, and (2) each method yielded a slightly different best focus position. 


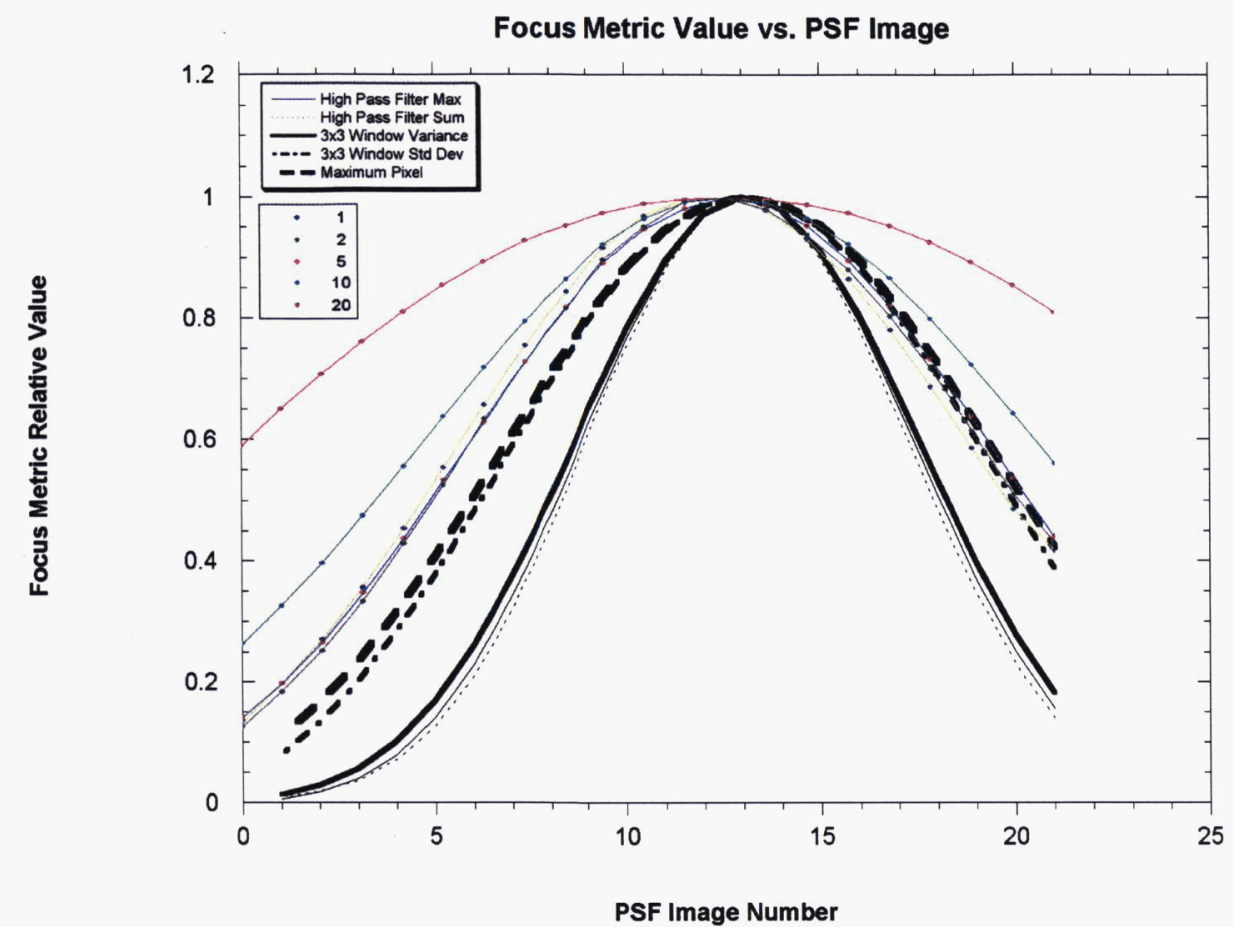

Figure 3. Comparison of methods for determination of best focus for NIRCam short wavelength channel. Encircled energy curves are indicated in color.

Encircled Energy results are shown in Figure 4 for noise- free PSF data for all the instruments. Peak pixel results for all the instruments are shown in Figure 5. 


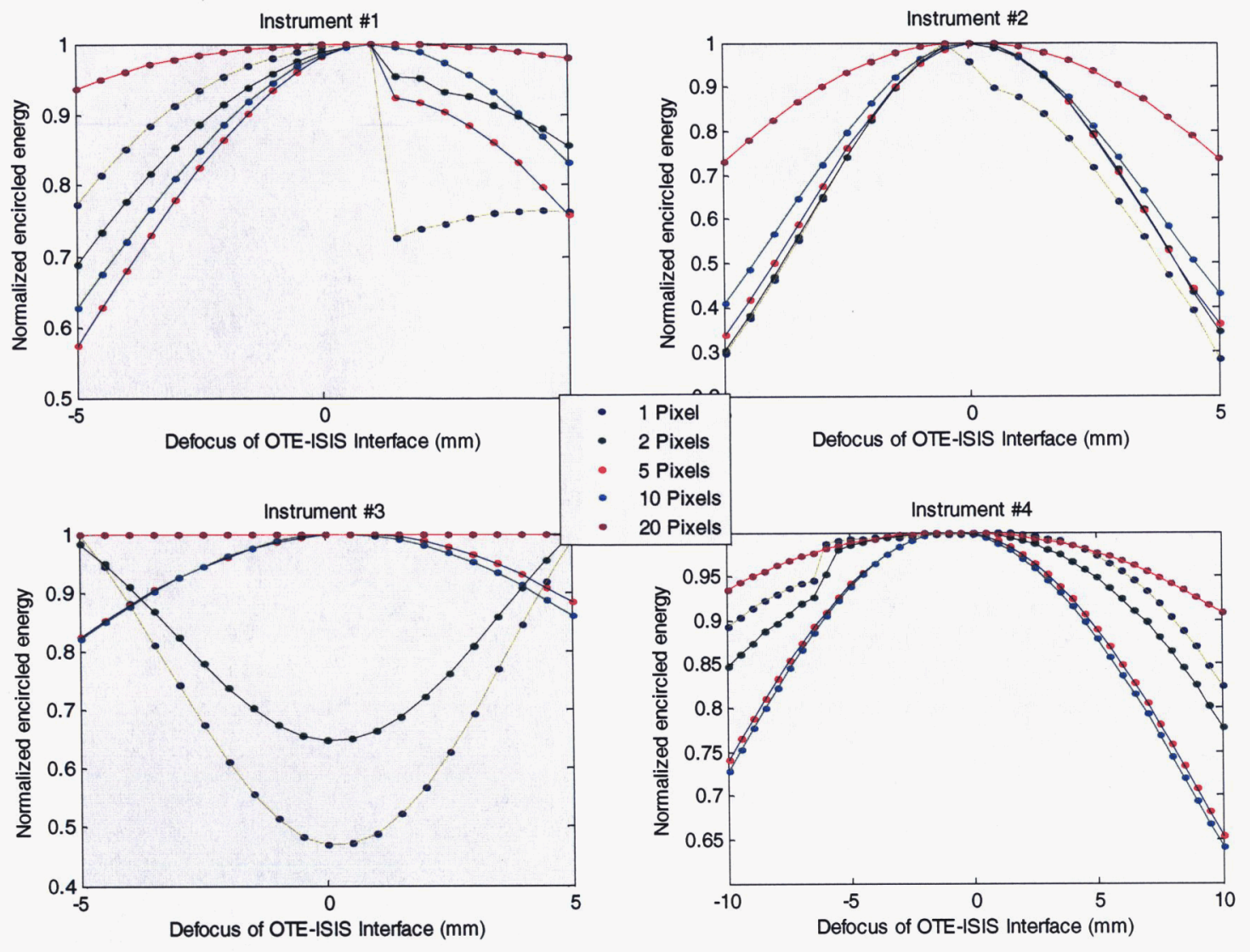

Figure 4. Encircled energy results for each model. In the figure, Instrument \#1 = NIRCam, Instrument \#2 = NIRCam short wavelength channel, Instrument \#3 = NIRSpec, Instument \#4 = MIRI. Curves are plotted for encircled energy within a circle with a diameter of $1,2,5,10$, and 20 pixels. 

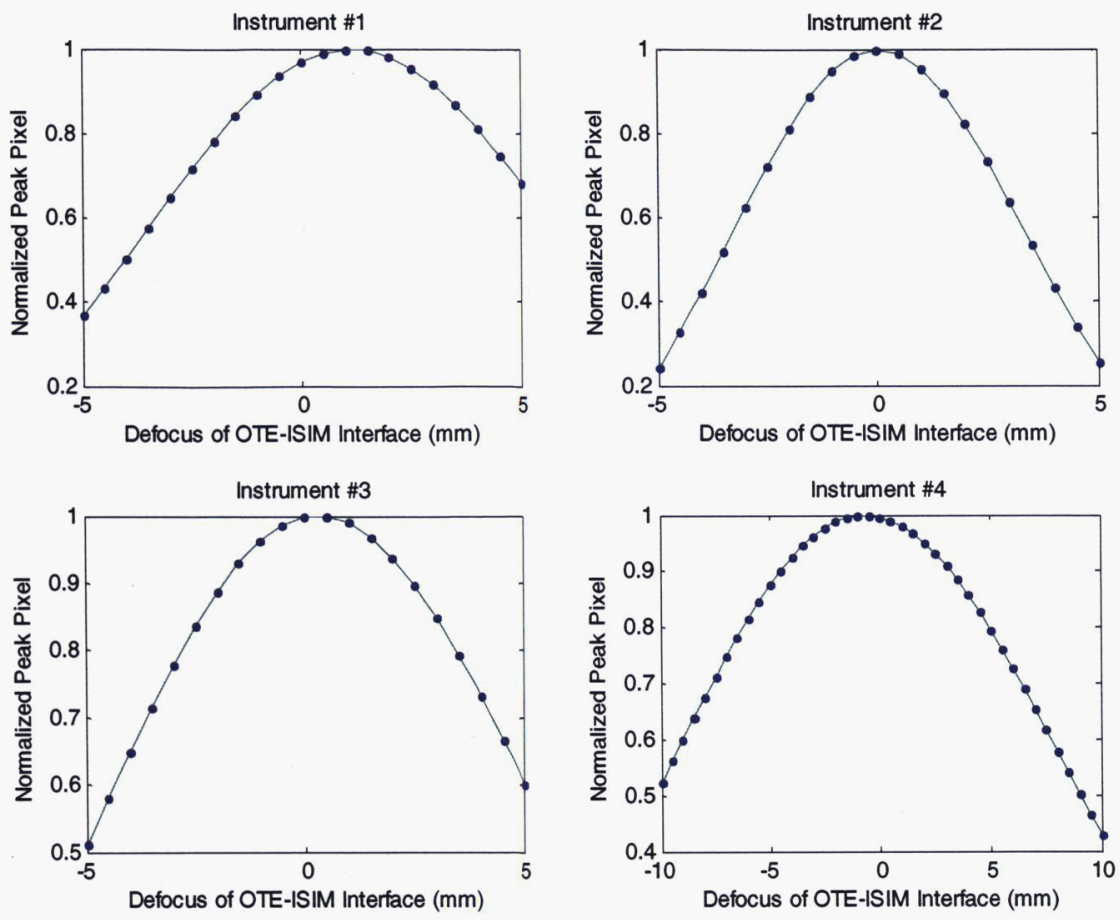

Figure 5. Results for peak pixel method, noise free data. In the figure, Instrument \#1 = NIRCam long wavelength channel, Instrument \#2 = NIRCam short wavelength channel, Instrument \#3 = NIRSpec, Instrument \#4 = MIRI. Curves are shown for encircled energy within a circle with a diameter of $1,2,5,10$, and 20 pixels.
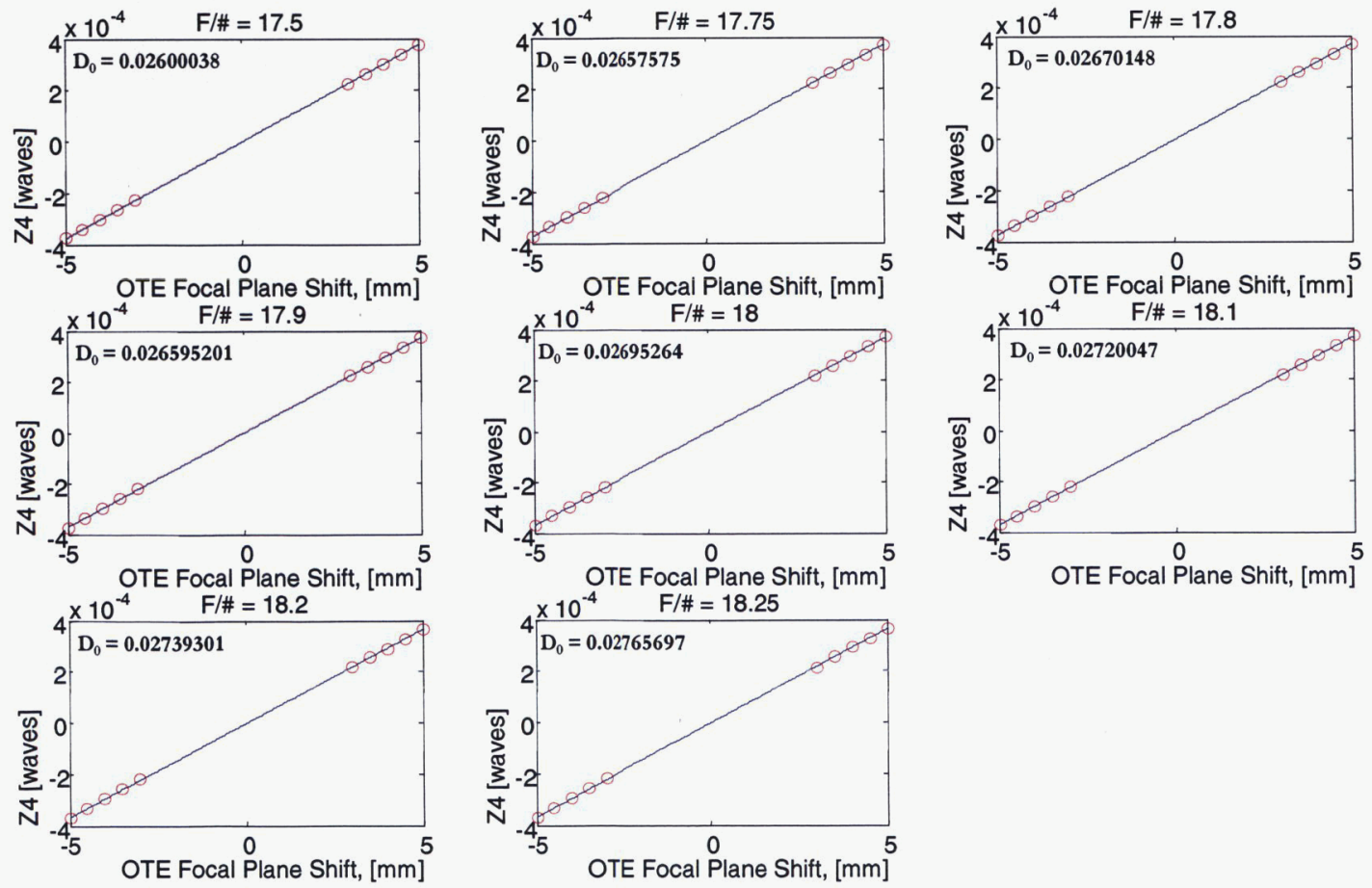

Figure 6. Results for phase retrieval method for noise free data in the NIRCam short wave channel. The plots show the recovered Zernike focus term, Z4, in waves, versus the amount of defocus at the OTE focal plane. Note that for each plot the value obtained for the best focus in $\mathrm{mm}$ is indicated by $\mathrm{D}_{0}$. 
Results obtained from the phase retrieval method are shown in Figure 6. In this figure, the plots show the Zernike focus term (Z4) retrieved from the algorithm for each OTE defocus value. Different results are shown for different $f / \# s$ that are used to fit the data. The best focus is determined by fitting a straight line through the data and then determining the zero point intercept, $D_{0}$. Since the algorithm has more difficulty recovering the data for in-focus PSFs the data points close to the in-focus PSFs were omitted for the fit.

Table 4 summarizes the results obtained for all the focus metrics. Note that there is an offset between all the focus Table 4. Comparison of results for the different methods.

\begin{tabular}{|c|c|c|c|c|c|c|}
\hline CLEAN & $\begin{array}{l}\text { CodeV } \\
\text { Predict }\end{array}$ & Peak Pixel & Peak High Pass & High Pass Sum & $\begin{array}{l}\text { Encircled } \\
\text { Energy }\end{array}$ & $\begin{array}{l}\text { Phase } \\
\text { Retrieval }\end{array}$ \\
\hline NIRCam Short & 0.08782 & 0.0250 & 0.0246 & 0.024815 & 0.0005 & 0.027882 \\
\hline NIRCam Long & 1.64065 & 1.1161 & 1.113115 & 1.11196 & 0.9996 & 1.1833 \\
\hline NIRSpec & 0.12600 & 0.4995 & 0.299455 & 0.298155 & 0.4995 & \\
\hline MIRI & -0.56867 & -0.5011 & -0.72035 & -0.70393 & -0.9991 & \\
\hline \multicolumn{7}{|l|}{ NoIsY } \\
\hline NIRCam Short & & 0.0250 & -0.017275 & 0.003585 & 0.0005 & 0.020878 \\
\hline NIRCam Long & & 1.1061 & 1.09105 & 1.107615 & 1.0006 & 0.37675 \\
\hline NIRSpec & & 0.4995 & 0.276455 & 0.2764 & 0.4995 & \\
\hline MIRI & & -0.9991 & -0.82428 & -0.80286 & -0.5011 & \\
\hline \multicolumn{7}{|l|}{ NOISIER } \\
\hline NIRCam Short & & 0.025025 & 0.55507 & 0.338745 & -0.0005 & 0.39725 \\
\hline NIRCam Long & & 1.05605 & 1.75718 & 0.95281 & 1.4996 & 0.044244 \\
\hline NIRSpec & & 0.4995 & 0.501485 & 0.50049 & -2.4997 & \\
\hline MIRI & & -0.9991 & -0.995725 & -0.9949 & -0.5011 & \\
\hline \multicolumn{7}{|l|}{ NOISTEST } \\
\hline NIRCam Short & & 0.065065 & -1.33646 & 2.49177 & -0.4995 & 0.00032164 \\
\hline NIRCam Long & & 0.9559 & 2.418665 & 0.47689 & -3.4998 & -0.27119 \\
\hline NIRSpec & & 0.9996 & -0.946445 & 0.017325 & -3.4998 & \\
\hline MIRI & & -1.9992 & -2.00969 & -0.864825 & -0.9991 & \\
\hline
\end{tabular}

metrics and the CodeV prediction. The Code $\mathrm{V}$ predicted position is where the rms wavefront error is calculated to be at a minimum. It is well known that this value is not the same as the $\mathrm{ZA}=0$ focus position for a system with aberrations.

\section{REFERENCES}

\footnotetext{
${ }^{1}$ S.K. Park and R.A. Schowengerdt, "Image Reconstruction by Parametric Cubic Convolution", Computer Vision, Graphics, and Image Processing 23, 258-272, 1983.

${ }^{2}$ M.W. Fitzmaurice, Kong Q. Ha, Chi Le, and Joseph M. Howard, End-to-end performance modeling of the James Webb Space Telescope (JWST) Observatory, Proc. SPIE 5867, 2005.
}

3 J.H. Price and D. A. Gough, "Comparison of Phase-Contrast and Fluorescence Digital Autofocus for Scanning Microscopy", Cytometry, V. 16, 283-297, (1994).

4 B.J. Bos, "Design, Calibration, and Operation of Mars Lander Camera, University of Arizona Ph.D. dissertation, 215238, 2002.

5 J.R. Fienup, J.C. Marron, T.J. Schulz, J. H. Seldin, "Hubble Space Telescope characterized by using phase-retrieval algorithms," Appl. Opt., 32, 1747-1767, (1993).

J. E. Krist and C. J. Burrows, "Phase-retrieval analysis of pre and post-repair Hubble Space Telescope images," Appl. Opt. 34, 4951-4964 (1995). 
R. Lyon, P.E. Miller, A. Grusczak, "Hubble Space Telescope Phase Retrieval: A Parameter Estimation," SPIE, Vol. 1567, (1991).

R. Lyon, J. Dorband, J. Hollis, "Hubble Space Telescope Faint Object Camera Calculated Point-Spread Functions,” Applied Optics, 36, 1752-1775, (1997).

C. Roddier, F. Roddier, "Combined Approach to the Hubble Space Telescope Wave-Front Distortion Analysis," Appl. Opt., 32, 2992-3008 (1993).

${ }^{6}$ D. S. Acton, T. Towell, J. Schwenker, J. Swensen, D.Shields, E. Sabatke, L. Klingemann, A.R. Contos, B. Bauer, K. Hansen, P.D. Atcheson, D. Redding, F. Shi, S. Basinger, B. Dean, L. Burns, "Demonstration of the James Webb Space Telescope Commissioning on the JWST Testbed Telescope," SPIE, these proceedings, (2006).

${ }^{7}$ B. H. Dean, D. S. Acton, D. Aronstein, S. Smith, R. Shiri, "Performance of fine-phasing algorithms on the James Webb Space Telescope Testbed," SPIE, these proceedings, (2006).

${ }^{8}$ R.W. Gerchberg and W.O. Saxton, "A Practical Algorithm for the Determination of Phase from Image and Diffraction Plane Pictures," OPTIK, 35, 237-246 (1972).

R. W. Gerchberg and W.O. Saxton, "Computer Techniques for Image Processing in Electron Microscopy," in Advances in Electronics and Electron Physics, Supplement 10, L Marton and C. Marton, eds. , (Academic Press, New York, NY, 1978).

N. C. Gallagher, B. Liu, "Method for Computing Kineforms that Reduces Image-Reconstruction Error," Appl. Optics 12(10) 2328-2335 (1973).

N. C. Gallagher, B. Liu, "Method for Computing Kineforms that Reduces Image-Reconstruction Error," Appl. Optics 12(10) 2328-2335 (1973).

R. A. Gonsalves, R. Dumais, P. Considine, “On Optimal Holographic Filters,” Proc. SPIE, Vol. 45, 1974.

${ }^{9}$ R. A. Gonsalves, P. Considine, "Phase-Retrieval from Modulus Data," J. Opt. Soc. Am., 66, 961-964 (1976).

${ }^{10}$ W. H. Southwell, "Wavefront Analyzer using a Maximum Likelihood Algorithm,” J. Opt. Soc. Am. A3, 396-399(1977).

11 Press, W. H., Teukolsky, S. A., Vetterling, W. T., and Flannery, B. P., Numerical Recipes in C, Cambridge University Press, second edition, (1992). 